\title{
Los profesores de inglés y su práctica docente: Un estudio de caso de los colegios fiscales de la ciudad de Cuenca, Ecuador
}

\author{
Ana M. Calle ${ }^{1}$, Susana Calle ${ }^{2}$, Juanita Argudo ${ }^{2}$,Eulalia Moscoso ${ }^{3}$, Andrew Smith ${ }^{3}$, Patricio \\ Cabrera $^{4}$ \\ ${ }^{1}$ CONCELT, Escuela de Lengua y Literatura Inglesa, Facultad de Filosofía, Universidad de Cuenca, \\ Cuenca, Ecuador. \\ ${ }^{2}$ CONCELT, Departamento de Idiomas, Facultad de Filosofía, Universidad de Cuenca, Cuenca, \\ Ecuador. \\ ${ }^{3}$ Departamento de Idiomas, Facultad de Filosofía, Universidad de Cuenca, Cuenca, Ecuador. \\ ${ }^{4}$ Estudiante egresado de la Escuela de Lengua y Literatura Inglesa, Facultad de Filosofía, Universidad \\ de Cuenca, Cuenca, Ecuador.
}

Autor para correspondencia: ana.calle@ucuenca.edu.ec

Fecha de recepción: 2 de agosto 2012 - Fecha de aceptación: 7 de noviembre 2012

\begin{abstract}
RESUMEN
Generalmente se cree que la principal razón para el limitado conocimiento y uso del inglés por parte de los estudiantes que se gradúan de los colegios está relacionada con la ausencia de estrategias efectivas de enseñanza, situación que ocurre en un alto número de colegios fiscales de la ciudad de Cuenca. Para conocer la medida en que se produce este hecho y para identificar las razones de los bajos conocimientos del inglés de estos estudiantes, se llevó a cabo un estudio exploratorio. La investigación incluyó una encuesta impresa con un cuestionario, la que fue contestada por 168 profesores de inglés, 92 profesores fueron monitoreados en el aula durante una clase, y finalmente 54 profesores fueron entrevistados de una población total de 215 profesores de inglés de los colegios fiscales de Cuenca. Los resultados revelan que el bajo rendimiento de los estudiantes de los colegios secundarios en el inglés se afecta negativamente debido al uso de estrategias de enseñanza tradicionales, como son el aprendizaje centrado en el profesor, la falta de interacción entre los estudiantes en la lengua meta y la confusión de los profesores al tratar de emplear diferentes estrategias comunicativas. En un futuro cercano, los resultados de la investigación se utilizarán para elaborar estrategias basadas en tareas y mejorar la habilidad de los profesores en la enseñanza de inglés a través de talleres de capacitación.
\end{abstract}

Palabras clave: Inglés, desempeño del profesor, estrategias de enseñanza, encuesta tipo cuestionario, monitoreo a los profesores, entrevistas.

\begin{abstract}
It is generally believed that the major reason for the limited knowledge and poor performance in English of the students graduating from high school is related to the absence and the lack of use of effective strategies of the English language teachers, and this in most of the public schools in Cuenca city. To check the extent and to identify the reasons of the low English literacy performance of graduating high school students an exploratory study was conducted. The research encompassed a paper questionnaire-based survey in which 168 English language teachers participated, 92 teachers were monitored in classroom during an English language class, and finally 54 teachers were interviewed, out of a total population of 215 English language teachers of the public schools of Cuenca city. Findings revealed that the poor English literacy performance of high school students is negative affected by the use of traditional teaching strategies, the teacher-centered approach, the lack of interaction with and among students in the target language, and the confusion of teachers when applying different communicative strategies. In the near future research results will be used to draft
\end{abstract}


task-based teaching strategies and to upgrade the teaching capacity of English language teachers via in-service workshops.

Keywords: English language, teacher performance, teaching strategies, questionnaire-based survey, teacher monitoring, interviews.

\section{INTRODUCCIÓN}

De acuerdo a Luo (2007) es indudable que el idioma inglés se ha convertido en lengua franca en el mundo actual; es decir, es el idioma que sirve como medio de comunicación entre las personas cuya primera lengua no es el inglés. Según este autor la globalización ha permitido al inglés ser el idioma internacional de la actualidad y progresivamente ha llegado a ser "una herramienta de conocimiento de las ciencias y las humanidades". El Ministerio de Educación del Ecuador, consciente de esta realidad, inició una reforma curricular en el año 1993 llamada proyecto CRADLE $^{1}$ aplicada a los colegios del país; sin embargo, el bajo rendimiento de los estudiantes persiste y, en el nivel universitario, los estudiantes no han logrado ingresar en niveles intermedios o avanzados de inglés. Ello se debe a múltiples factores que caracterizan la enseñanza de inglés en el Ecuador en los que se desataca el uso de estrategias inadecuadas en las aulas de clase.

Los profesores fiscales de inglés no emplean estrategias que fortalezcan la competencia comunicativa, pues continúan utilizando estrategias tradicionales que obstaculizan el desarrollo de dicha competencia, así como también de las destrezas lingüísticas (leer, escribir, escuchar y hablar) lo que repercute en el bajo rendimiento estudiantil. De acuerdo a un estudio aplicado, a cinco colegios fiscales de la ciudad de Cuenca, en el que se empleó una prueba de inglés, los bachilleres obtuvieron un promedio de 10,3 sobre 20 puntos (CONCELT $\left.{ }^{2}, 2004\right)$. A nivel nacional se mantiene todavía una tradición en la enseñanza de inglés que enfatiza el aspecto gramatical circunscrito a la instrucción pasiva de reglas y formas (Ministerio de Educación, 2008). CONCELT (2004), indica que en Cuenca los profesores fiscales se centran en la gramática y no utilizan estrategias modernas que desarrollen la lecto-escritura siguiendo la tendencia nacional según Vargas (1995). Observaciones a profesores de inglés del cantón Cuenca realizadas por personeros del Departamento de Idiomas Extranjeros de la Dirección de Estudios de la provincia del Azuay en el año 2008 (Arévalo, 2009), indican el énfasis en las formas lingüísticas y una resistencia de los profesores fiscales a cualquier tipo de innovación.

Al existir un nuevo currículo (Proyecto CRADLE) en la educación media, acorde con estrategias de enseñanza moderna, se hace pertinente examinar las prácticas docentes de los profesores fiscales de inglés de los colegios para determinar debilidades e implementar alternativas. Frente a esta situación se plantea la siguiente interrogante: ¿Qué sustento pedagógico fundamenta las estrategias de enseñanza que se utilizan frecuentemente en los colegios fiscales de la ciudad de Cuenca en la asignatura de inglés? Con la respuesta a esta interrogante, este proyecto identifica las estrategias de enseñanza empleadas por los profesores de inglés para en un futuro proponer alternativas de mejora. Se conduce, así, una investigación exploratoria mixta, cualitativa y cuantitativa.

La literatura de esta problemática abarca tres ámbitos de la enseñanza: un aspecto teórico sobre la relevancia de la enseñanza comunicativa de un idioma extranjero $\left(\mathrm{CLT}^{3}\right)$, un análisis de las políticas y prácticas docentes utilizadas en la enseñanza del idioma inglés en la actualidad en algunos países incluido el Ecuador, particularmente en la ciudad de Cuenca, y finalmente, experiencias y percepciones de los profesores en la enseñanza de inglés.

\footnotetext{
${ }^{1}$ Reforma Curricular para el mejoramiento de la enseñanza-aprendizaje de inglés (Curriculum Reform Aimed at the Development of the Learning of English).

${ }^{2}$ Centro de consultoría para la enseñanza de inglés de la Facultad de Filosofía de la Universidad de Cuenca.

${ }^{3}$ CLT: Communicative Language Teaching.
} 


\subsection{La enseñanza comunicativa}

A lo largo de la historia de la enseñanza de una segunda lengua (SL) o lengua extranjera (LE) han existido una serie de enfoques metodológicos fundamentados en diferentes escuelas psicológicas. El método de Gramática y Traducción y el método Audio Lingual fundamentados en el conductismo se han dedicado a la enseñanza de estructuras gramaticales aisladas y a la traducción; otras estrategias metodológicas modernas, basadas en el cognitivismo y englobadas en lo que se ha llamado la Enseñanza Comunicativa del Lenguaje, se han enfocado en la comunicación como fin y medio del aprendizaje de un idioma, así como en el desarrollo de la competencia comunicativa en los aprendices de una segunda lengua (Alcón, 2004). Para alcanzar esta competencia comunicativa, se utiliza una serie de estrategias de enseñanza-aprendizaje que, de acuerdo a Richards y Rodgers (2001), son ilimitadas. Estas estrategias incluyen actividades que requieren compartir información, negociar los significados e interactuar en la lengua extranjera. Las actividades de clase toman distintas formas y se concentran en completar tareas utilizando el idioma extranjero. La mayoría de ellas se desarrollan a través de proveer cierta información a un estudiante y ocultar la misma información a otro alumno, de manera que se produce una interacción en la que uno de ellos trata de descubrir la información desconocida. Así, la conversación se centra en la transmisión de la información más no en elementos aislados de la lengua como ocurre en los enfoques estructurales de Gramática y Traducción y el método Audio Lingual. El centro del proceso enseñanza-aprendizaje es el alumno. De allí que Long, citado por Alcón (2004), considera que "el nivel de éxito en el aprendizaje de la lengua depende de la calidad de interacción" que se provea en el aula de clase. De otra parte, Littlewood, citado por Richards y Rodgers (2001), distingue dos tipos de actividades o estrategias comunicativas: funcionales y sociales. Las primeras incluyen actividades tales como comparar un grupo de dibujos y determinar similitudes y diferencias; descifrar una secuencia de un relato a través de poner en orden ilustraciones del relato; brindar instrucciones a otro estudiante para que realice un dibujo determinado; seguir instrucciones y resolver problemas en base a pistas. Actividades de interacción social incluyen sesiones de conversaciones y discusiones, dramatizaciones cortas, improvisaciones y debates. Por otro lado, las estrategias utilizadas por el enfoque tradicional estructural son limitadas y se centran en la repetición de oraciones aisladas y en el análisis de estructuras gramaticales. Larsen-Freeman, citada por Liu (2004), señala que la discrepancia entre estos dos enfoques, el estructural y el comunicativo, ha influenciado en los profesores de lenguas en decidirse por una u otra tendencia.

En palabras de Alcón (2004) “... parece existir un dilema entre la enseñanza de la lengua enfocada en el análisis de las estructuras versus una enseñanza centrada en la experiencia, sin un énfasis estructural". El presente estudio, considera a la enseñanza tradicional, desde la perspectiva de Alcón (2004), como el desarrollo del "conocimiento formal de las reglas gramaticales", contrastando con lo comunicativo como "el resultado del uso natural de la lengua". Por lo tanto, el método audio lingual que hacía uso de ejercicios de repetición de estructuras aisladas sin un contexto significativo, memorización de oraciones, frases, y análisis de las estructuras, no desarrollaba una competencia comunicativa (Richards and Rodgers, 2001). Cabe insistir que estos tres aspectos mencionados por Richards y Rodgers constituyen, para los autores del presente estudio, el concepto de la enseñanza tradicional al que se hace referencia a lo largo del documento.

\subsection{Políticas y prácticas docentes}

La tendencia comunicativa se evidencia en la implementación de nuevos currículos en algunos países, donde el inglés no es la primera lengua, tal es el caso de China (Luo, 2007), Taiwán (Wang, 2008), República Dominicana (Garrido, 2007) y el Ecuador. China empezó en el año 2004 ejecutando una reforma en la enseñanza de inglés en el nivel superior que enfatiza el desarrollo de la competencia comunicativa alejada de un conocimiento único en la forma lingüística y centrada en el desarrollo de todas las destrezas del lenguaje. Esto ha significado una enseñanza centrada en el alumno que ha desarrollado su autonomía en base a la utilización de procesos individualizados, colaborativos y de hipertextos basados en el uso de la computadora (Luo, 2007). Así mismo, en el año 2004 el gobierno de la República Dominicana promueve un estudio piloto de dos instituciones educativas particulares, conocidas por sus resultados positivos en la enseñanza del inglés como lengua extranjera, para ser tomadas como ejemplo en una implementación curricular en el nivel público. Las dos instituciones se enmarcan en un enfoque comunicativo del lenguaje. Una de las escuelas utiliza un programa de 
inmersión del inglés al dictar casi todas las materias en este idioma; la otra institución educativa dicta inglés una hora diaria a sus estudiantes en base a un programa de literatura en inglés. Los estudiantes de las dos escuelas han desarrollado destrezas comunicativas, sin embargo, los estudiantes de la escuela de inmersión presentan destrezas superiores (Garrido, 2007). La autora concluye que los aprendices pueden obtener grandes beneficios al "analizar, deducir, y pensar en la lengua extranjera, mientras al mismo tiempo aprenden contenidos de una materia en Inglés". Taiwán es otro país cuyo Ministerio de Educación estandarizó la enseñanza del inglés desde el tercer grado de primaria (9 años) con un enfoque comunicativo en el año 2005 (Wang, 2008). Taylor y Mulhall (2001) informan sobre los resultados positivos de un estudio realizado en Tanzania, Sri Lanka, India y Etiopía en el que los profesores primarios del área rural relacionan el currículo nacional con las experiencias de los estudiantes, especialmente con la agricultura; es decir, sugieren contextualizar la enseñanza.

Pensamos que estas consideraciones son importantes en la enseñanza de una lengua extranjera. El Proyecto CRADLE, como se le llamó a la reforma curricular de la enseñanza de inglés, implementado en el Ecuador en el año 1993, responde a esta contextualización con la creación de una serie de 6 textos de enseñanza llamados Our World Through English (OWTE) y como su nombre indica, presenta contenidos que describen la realidad del país por medio del inglés. Acorde con los nuevos enfoques de la enseñanza de una segunda lengua o de una lengua extranjera, "los gobiernos Ecuatoriano y Británico firmaron en el año 1992 un convenio de cooperación técnica bilateral con el objetivo principal de mejorar la calidad de la enseñanza-aprendizaje del idioma Inglés en el país para lo cual se inició la reforma curricular del área" (Ministerio de Educación y Culturas del Ecuador, 2009). El proyecto CRADLE, entre otros resultados, ha logrado lo siguiente: "producción de la primera edición de seis libros de la serie de textos OWTE escritos y publicados localmente con guías didácticas para el profesor, material auditivo de apoyo y material de exámenes y pruebas producidos y utilizados aproximadamente por 1.200.000 estudiantes y sus profesores en los años escolares de 19934 a 1998-9" (Ministerio de Educación y Culturas del Ecuador, 2009). También se ha capacitado a 5.000 profesores y se ha establecido las bases legales para la implementación del nuevo currículo a lo largo de todo el país. Los colegios fiscales y fiscomisionales están obligados a incluir 5 horas de inglés semanal en sus establecimientos en los octavos, novenos y décimos grados de educación básica y en todo el bachillerato, así como a usar el material didáctico de la serie OWTE (El Mercurio, 2006). De acuerdo a datos del Ministerio de Educación, el rendimiento de los estudiantes ha mejorado de acuerdo a un examen nacional aplicado a los estudiantes ecuatorianos con un promedio de 15 sobre 20 hasta la fecha. Más adelante analizaremos las realidades locales de los colegios de Cuenca.

Sin embargo, a pesar de esta serie de innovaciones en diferentes países, se ha observado problemas en la implementación de los nuevos currículos. Wang (2008) indaga los problemas que surgieron al aplicarse el currículo del año 2004 en Taiwán. Este autor observó difícultades en las prácticas docentes al aplicar los nuevos textos e indica que un $62 \%$ de profesores entrevistados informaron que utilizan un enfoque comunicativo y un $11 \%$ prefiere la gramática y la traducción, a pesar de ser el enfoque comunicativo obligatorio. De acuerdo a Liyianage (2004) en Sri Lanka el Ministerio de Educación en colaboración con agencias extranjeras, desarrollaron textos y materiales para ser empleados por profesores en todo el país, pero no se han registrado resultados positivos. Liyanage (2004) señala que los materiales no concuerdan con las estrategias de alumnos y preferencias pedagógicas de profesores, estrategias que incluyen procesos de deducción y procesos centrados en el profesor. Así mismo, otro estudio realizado por Mohamed (2006) en las islas Maldivas señala la resistencia al cambio por parte de los profesores de inglés, a pesar de la implementación de nuevos materiales y currículos. Esta autora señala que "los métodos de enseñanza y la dinámica de la clase no han cambiado. En estas clases el profesor fue el centro del aprendizaje, el contenido fue dominado por elementos gramaticales y el modelo de instrucción fue claramente el de transmisión". De acuerdo a las observaciones de Thornbury, citado por Mohamed (2006), los profesores no se han desviado de los enfoques tradicionales basados en la gramática; los enfoques alternativos no han influenciado en la práctica de la enseñanza de inglés. Por lo tanto, la práctica docente no ha sido afectada por el desarrollo teórico actual. Se puede observar una serie de obstáculos para aplicar la enseñanza comunicativa del inglés y, en palabras de Savignon y Wang (2003), no sorprende observar como las innovaciones del inglés en contextos extranjeros basadas en un enfoque comunicativo han enfrentado 
una serie de retos. A este respecto, se hace también necesario indagar las experiencias y preparación de los profesores como elementos constitutivos de esos retos.

\subsection{Experiencias docentes}

Según Mohamed (2006), estudios realizados por Burns, Kumaravadivelu y Nunan, sugieren que aunque muchos profesores manifiesten adhesión con la enseñanza comunicativa, los principios de esta enseñanza son raramente aplicados en el aula. De acuerdo a un estudio llevado a cabo por Liu (2004) en el que se entrevistó a profesores de inglés de todo el mundo, se encontró que el método de gramática y traducción sigue siendo utilizado en muchas partes del mundo, particularmente en países donde el inglés es una lengua extranjera y principalmente donde existen amplios grupos de estudiantes por clase con niveles bajos de suficiencia en la lengua. Es interesante notar las inconsistencias de la teoría con la práctica, ya que a pesar de las bajas preferencias por metodologías estructurales, un $75 \%$ de profesores no nativo hablantes del inglés señalaron que utilizan el método de gramática y traducción para desarrollar la escritura y la lectura. Este estudio concluye que esta práctica docente podría ser ocasionada por el medio lingüístico en el que no se usa la lengua extranjera, lo que no permite mucha exposición a esta lengua ni a materiales auténticos. Otra conclusión señala que los profesores tienen resistencia a cualquier práctica innovadora de enseñanza debido a su baja competencia comunicativa en la lengua, al gran tamaño de grupos de estudiantes en sus clases, y a las evaluaciones centralizadas, entre otros factores. Por lo tanto, es necesario analizar la variable relacionada con el conocimiento y preparación de los profesores.

Concordamos con McLaughlin (1996), cuando señala que "el factor más importante para mejorar la calidad de la educación en países en vías de desarrollo parece ser el incremento de mejores profesionales de la educación". Shono (2004) confirma esta idea al indicar que los programas que forman profesores de inglés tienen gran responsabilidad en el entrenamiento teórico y práctico de lo que implica ser un buen profesor de lenguas. En la misma línea, Vargas (1995), de acuerdo a un estudio previo a la implementación del proyecto CRADLE, señala que los bajos niveles de aprendizaje de inglés en el Ecuador pueden deberse, entre otros factores, a un sílabo y metodología obsoletos centrados en el profesor y enfocados en la gramática y traducción, y a profesores carentes de una apropiada formación, pues en las observaciones de clase realizadas, se notó la tendencia a usar la lengua materna como medio de instrucción y el idioma extranjero se presentó de una manera descontextualizada. Ilustrando esta situación, el Ministerio de Educación y Cultura del Ecuador (1998) ha señalado que "el programa de inglés consistía en una lista larga de estructuras gramaticales, y que muchos profesores repetían el programa del primer año durante los seis años, porque consideraban que los estudiantes no habían aprendido el verbo to be”. Es así que la implementación de un sílabo estructural no solamente determina los contenidos a ser enseñados, sino también la metodología a ser aplicada en el aula.

Es importante analizar algunas realidades locales. Criollo y Pulla (2000) en un estudio elaborado en Cuenca, Ecuador, señalan que el $41 \%$ de los profesores fiscales tienen un título en inglés, mientras que el $16 \%$ tienen un título de bachiller, el resto tiene título en otras especialidades. Según un estudio realizado por CONCELT (2004) de la Universidad de Cuenca, los profesores no utilizan e incluso desconocen metodologías modernas, especialmente en lo que respecta a la enseñanza de la lectura y particularmente de la escritura. Además se enfatiza en la enseñanza de la gramática, ya sea de manera tradicional o comunicativa; estos datos coinciden con los expuestos por Liu (2004). A través de observaciones de clase, Criollo y Pulla (2000) pudieron corroborar que el uso del inglés por parte de los profesores es mínimo en la educación pública de Cuenca. Observaciones a profesores de inglés del cantón Cuenca realizadas por personeros del Departamento de Idiomas Extranjeros de la Dirección de Estudios de la provincia del Azuay en el año 2008 (Arévalo, 2009) señalan que existe una resistencia de los profesores fiscales a cualquier tipo de innovación, el inglés casi no es empleado por los profesores y se enfatiza en la explicación de formas lingüísticas antes que en el uso de las mismas. Estas situaciones se asemejan a los reportes de estudios internacionales antes mencionados y que podrían haber incidido en el desarrollo del inglés de los estudiantes.

Este bajo dominio del idioma se ha evidenciado en el rendimiento estudiantil de bachilleres fiscales de Cuenca. Así, en una prueba aplicada a tres colegios particulares, el promedio del 
rendimiento estudiantil fue de 16,5 puntos sobre 20 , mientras que los resultados arrojados por la misma prueba aplicada a estudiantes de cinco colegios fiscales fue de 10,3 (CONCELT, 2004).

En conclusión, la enseñanza tradicional del inglés en el Ecuador ha sido superada en gran medida por la implementación del Proyecto CRADLE que ha contribuido de manera significativa a un mejoramiento en términos de currículo. De acuerdo a Criollo y Pulla (2000), antes del proyecto CRADLE, los materiales utilizados en la enseñanza del inglés respondían a una metodología estructural que enfatizaba la gramática. Estas autoras indican que el proyecto CRADLE, sin duda, se constituye en un paso adelante desde un sílabo estructural hacia un sílabo más procedimental que sigue el enfoque natural planteado por Krashen y Terrell (1983), el cual se enmarca en la enseñanza comunicativa. Esta situación coincide con la información oficial que señala que "los resultados indican el éxito del programa en llevar el proceso de enseñanza-aprendizaje hacia un marco comunicativo dejando atrás una estéril orientación estructural" (Ministerio de Educación y Culturas del Ecuador, 1998). Este desarrollo en la enseñanza del inglés en el Ecuador coincide con el desarrollo de enseñanza ocurrido en otras partes del mundo. A pesar de los logros alcanzados en el país, pensamos que existe un largo camino por recorrer y que puede estar centrado, entre otros factores, en las estrategias de enseñanza de nuestros profesores fiscales, pues los datos aquí expuestos nos señalan esta dirección. Vale mencionar que no se cuenta con publicaciones que brinden información sobre la realidad actual de la enseñanza de inglés en el área urbana de Cuenca. Se hace relevante, pues, investigar al respecto. Por lo tanto, los objetivos de este estudio se dirigen a determinar los tipos de estrategias de enseñanza que están siendo empleados por los profesores fiscales de inglés en los colegios públicos.

\section{MATERIALES Y MÉTODOS}

La tendencia nacional en la enseñanza del inglés, de la que Cuenca no se exime, se ha dirigido hacia una instrucción tradicional que se manifiesta en el bajo rendimiento estudiantil durante las dos últimas décadas. De este problema se deriva una interrogante que hace referencia a la identificación del sustento pedagógico que fundamenta las estrategias de enseñanza utilizadas frecuentemente en los colegios fiscales en la asignatura de inglés. Diferentes metodologías se aplican para resolver la pregunta de investigación mencionada. Se emplea una metodología de investigación de tipo exploratorio mixto, cualitativo y cuantitativo (Grotjahn, citado por Mackey y Gass, 2005)

La metodología exploratoria trata de identificar los componentes de un fenómeno educativo a fin de determinar la situación real de la enseñanza de inglés en los colegios fiscales de la ciudad. Al no contar con fuentes bibliográficas actualizadas que registraran la situación de la enseñanza de inglés, se hace relevante explorar esta realidad enfocada a la metodología empleada por los docentes de inglés. La población investigada está compuesta por profesores fiscales de la asignatura de inglés de los colegios de la ciudad de Cuenca; es decir, profesores que tienen una partida fiscal emitida por el Ministerio de Educación y Cultura del Ecuador y laboran en colegios fiscales del área urbana, los mismos que suman un número de 215 docentes que constituye el universo de esta investigación siendo cada profesor la unidad de análisis. Los instrumentos utilizados en esta investigación fueron: una encuesta, una observación y una entrevista. La encuesta fue contestada por 168 profesores; de estos profesores 92 docentes accedieron a ser observados en una clase y de estos últimos, 54 fueron entrevistados.

En este estudio, la metodología exploratoria de carácter cuantitativo utiliza una encuesta diseñada por Brown y Rodgers (2004), que tiene el propósito de recolectar información sobre las concepciones de los profesores en relación a la enseñanza de una lengua. Esta encuesta es adaptada por el equipo de investigadores, traducida del inglés al español y piloteada por dos ocasiones a profesores de inglés del Departamento de Idiomas de la Universidad de Cuenca y profesores de colegios fiscales. Acogiendo las sugerencias del pilotaje, se hacen algunos ajustes que dan lugar a la encuesta final. Las encuestas son enviadas a los 215 profesores seleccionados, a través de la División de Idiomas Extranjeros de la Dirección Provincial de Educación del Azuay (DIEX). Sólo 168 profesores completan y entregan la encuesta. La encuesta incluye, en primer lugar, información socio-demográfica de los profesores, tales 
como: datos personales, titulación, experiencia docente, competencia en la lengua extranjera y capacitación en el área de inglés. En segundo lugar, este instrumento pretende determinar las concepciones de los profesores con respecto a la enseñanza de la lengua y contiene 19 preguntas enfocadas a criterios tradicionales así como también comunicativos sobre su enseñanza.

Respecto al tratamiento de los resultados de la encuesta, se realiza el siguiente procedimiento:

a) Se clasifica las preguntas según sus respuestas para que midan directamente la enseñanza tradicional o la no tradicional;

b) Las respuestas a cada pregunta cubren un rango ordinal de 1 (está completamente en desacuerdo) a 4 (está de acuerdo). Dependiendo de las preguntas, se asigna un porcentaje de tradicionalismo a las respuestas. Ante una pregunta sobre enseñanza tradicional con una contestación de 4 , se cataloga a la respuesta con un porcentaje de $100 \%$ tradicional; en cambio, ante una pregunta sobre enseñanza no tradicional o comunicativa en donde se conteste 4 , el porcentaje asignado sería de $0 \%$ de tradicionalismo. Por ejemplo, en la pregunta tradicional "La gramática es la parte más importante al aprender inglés", si la respuesta es 4 , significa un porcentaje de $100 \%$ tradicional. Por otro lado, si a la pregunta no tradicional "La comunicación real y frecuente le permitirá al estudiante mejorar su nivel de inglés", si responde con 4 , se obtiene un $0 \%$ de porcentaje tradicional; y

c) Al haberse incluido preguntas que determinaban diferentes grados tanto de enseñanza tradicional como de enseñanza comunicativa, se hace necesario fijar un orden de prelación de las mismas y relativizar sus valores en una escala de 1 a 3 , en cuanto a su importancia en medir lo tradicional o no tradicional de la educación. En otras palabras, el orden de prelación mostrará que hay preguntas que pueden caracterizar el tradicionalismo más claramente que otras debido a que son tres veces más determinantes que otras en lo tradicional. Esto significa que una pregunta con importancia 3 significó que su respuesta pesó el triple que una con importancia 1. Por ejemplo, la pregunta "la escritura de un párrafo debe ser evaluada considerando primordialmente la forma lingüística (gramática y puntuación)" se la valora con 1 punto, mientras que la pregunta "la repetición mecánica de estructuras aisladas es útil para la enseñanza de inglés" se la pondera con un valor de 3 puntos. Sobre la base de las modificaciones previas, se crea un índice de medición del grado de enseñanza tradicional empleada por el profesor denominado ATES (Average Traditional Education Score), el cual varía de 0 a 1, siendo cero un valor ideal de educación no tradicional, y uno $(100 \%)$ implicando una educación totalmente tradicional.

Como se mencionó, una observación in situ es otro instrumento utilizado. Se realiza a 92 docentes quienes accedieron, a través de un consentimiento informado, a recibir a los investigadores del proyecto en sus aulas de clase por una sola vez. Es importante indicar que en la gran mayoría de clases observadas, se pudo constatar la existencia de un número alto de estudiantes. Se puede encontrar detalles del número de alumnos al final de la Tabla 2. Dos investigadores, en forma conjunta, observan a un profesor y registran sus criterios en un formulario construido por el equipo de investigadores. El formulario apunta a recolectar información en las siguientes áreas: manejo de clase, estrategias de enseñanza, retroalimentación que usa el profesor y su dominio del inglés. Contiene preguntas cerradas, sin embargo, los observadores también podían incluir comentarios con respecto a las diferentes situaciones de clase. Este instrumento es diseñado para la observación de una hora de clase. Cada área observada se valora de acuerdo a tres parámetros: siempre, a veces y nunca. Los parámetros de observación de estas áreas están orientados a determinar el nivel de uso de didáctica tradicional en la hora de clase. Cabe señalar que la ejecución de la observación fue previamente comunicada a los docentes que iban a ser observados lo que podría limitar los resultados de este instrumento en tanto los profesores estaban preparados para la misma.

Un tercer instrumento empleado fue una entrevista que se llevó a cabo luego de la observación de clase. Solamente 54 profesores de los 92 observados accedieron a ser entrevistados. Para ejecutar esta técnica de investigación se elabora una guía de preguntas que apunta a corroborar lo observado en clase relacionado con las prácticas tradicionales así como también con estrategias comunicativas utilizadas en el aula. También este instrumento sirve para explorar las razones por las que los docentes empleaban determinadas técnicas de enseñanza. Debido a la condición cualitativa de la información obtenida por la entrevista, se procedió a codificar la información en el sistema ATLAS.ti, lo cual permitió realizar un análisis interpretativo y establecer tendencias de enseñanza. 


\section{RESULTADOS Y DISCUSIÓN}

La muestra de esta etapa exploratoria fue de 168 educadores, lo que correspondió casi al $80 \%$ del universo, que representa una muestra significativa; este número se definió a partir de aquellos docentes que completaron las encuestas pertenecientes a 29 colegios (Tabla 1). Comparando la proporción de docentes por establecimiento educativo de acuerdo al número total de docentes y el número de docentes encuestados, se obtiene la Figura 1.

Tabla 1. Composición de la población y muestra.

\begin{tabular}{lccccc}
\hline Parámetro & $\begin{array}{c}\text { Número total o } \\
\text { porcentaje }\end{array}$ & Promedio & $\begin{array}{c}\text { Desviación } \\
\text { estándar }\end{array}$ & Máximo & Mínimo \\
\hline $\begin{array}{l}\text { Número total de docentes } \\
\text { fiscales de inglés }\end{array}$ & 215 & & & \\
$\begin{array}{l}\text { Número de colegios fiscales } \\
\text { encuestados }\end{array}$ & 29 & & & & \\
$\begin{array}{l}\text { Número de profesores } \\
\text { encuestados }\end{array}$ & 168 & & & & \\
$\begin{array}{l}\text { Número de profesores por } \\
\text { colegio fiscal }\end{array}$ & & 6 & 3,85 & 19 & 2 \\
\hline
\end{tabular}

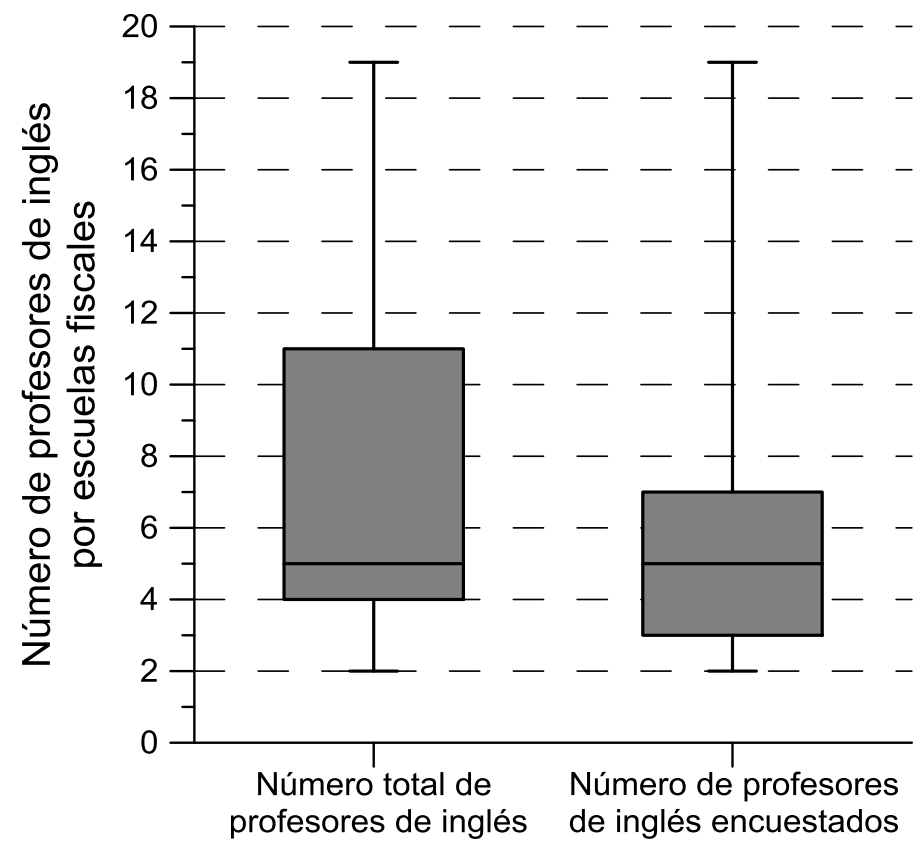

Figura 1. Variación del número total de profesores de inglés y número de profesores encuestados por colegio fiscal.

En la Figura 1 se presenta la muestra seleccionada de 29 planteles en relación al total de establecimientos fiscales de la ciudad de Cuenca. Existe un número máximo de 19 docentes por establecimiento cuya media es de 6 docentes. Ello significa que el $50 \%$ de la muestra presenta un número menor o igual a 5 . El cuartil inferior y superior de la población de docentes encuestados es la misma, lo cual indica la existencia uniforme de docentes encuestados. Asimismo, se evidencia que el número total de docentes presenta un cuartil mayor en la parte superior de la media, cuestión que no ocurre en la parte inferior de este mismo cuartil (con una variación de 4 y 5 docentes por cuartil), comparado con el total de docentes que superan la media por establecimiento (que varía de 5 a 11). En 
otras palabras, la distribución del número total de docentes en la parte superior no presenta uniformidad en relación a la parte inferior a la media. Si se considera el mínimo, el máximo, la media y toda la proporción de los cuartiles, se evidencia que el cuartil correspondiente a la muestra se encuentra mucho más proporcionado que el cuartil del total de la población. En consecuencia, la muestra es muy representativa a pesar de que el total de docentes presenta mucha variación.

Tabla 2. Características de la población de los profesores encuestados y número de estudiantes en colegios diurnos y nocturnos.

\begin{tabular}{|c|c|c|c|c|c|}
\hline Parámetro & $\begin{array}{l}\text { Número total } \\
\text { o porcentaje }\end{array}$ & Promedio & $\begin{array}{l}\text { Desviación } \\
\text { estándar }\end{array}$ & Máximo & Mínimo \\
\hline Edad de los profesores & & 44,25 & 10,74 & 71 & 25 \\
\hline \multicolumn{6}{|l|}{ Género de los profesores } \\
\hline $\begin{array}{r}\text { Masculino } \\
\text { Femenino }\end{array}$ & $\begin{array}{l}27 \% \\
73 \%\end{array}$ & $\begin{array}{l}47,19 \\
43,16\end{array}$ & & & \\
\hline Años de experiencia de & & 16,01 & 10,17 & 40 & 0,25 \\
\hline Masculino & $26 \%$ & 15,67 & & & \\
\hline Femenino & $74 \%$ & 16,35 & & & \\
\hline \multicolumn{6}{|c|}{$\begin{array}{l}\text { Estudios de pregrado de los docentes } \\
\text { encuestados }\end{array}$} \\
\hline Bachilleres de colegio & $6,01 \%$ & & & & \\
\hline Otras especialidades & $30,06 \%$ & & & & \\
\hline $\begin{array}{r}\text { Profesor de Segunda enseñanza } \\
\text { de inglés }\end{array}$ & $13,11 \%$ & & & & \\
\hline $\begin{array}{r}\text { Licenciados en Ciencias de la } \\
\text { Educación en inglés }\end{array}$ & $50,82 \%$ & & & & \\
\hline \multicolumn{6}{|c|}{$\begin{array}{l}\text { Estudios de postgrado de los docentes } \\
\text { encuestados }\end{array}$} \\
\hline $\begin{array}{r}\text { Postgrados en educación de } \\
\text { inglés }\end{array}$ & $0,00 \%$ & & & & \\
\hline Otros postgrados & $18,56 \%$ & & & & \\
\hline $\begin{array}{l}\text { Porcentaje de los profesores } \\
\text { que han vivido temporalmente } \\
\text { en un país de habla inglés }\end{array}$ & $28,66 \%$ & & & & \\
\hline $\begin{array}{l}\text { Número de estudiantes por } \\
\text { clase de colegios diurnos }\end{array}$ & & 35,94 & 7,81 & 50 & 9 \\
\hline $\begin{array}{l}\text { Número de estudiantes por } \\
\text { clase de colegios nocturnos }\end{array}$ & & 18,10 & 5,69 & 27 & 11 \\
\hline
\end{tabular}

El parámetro relacionado con la edad, nos indica que el promedio de edad de docentes sobrepasa los 43 años. Además, una mayoría de docentes, el 64,7\%, sobrepasa los 40 años. De estos datos, se puede inferir que los maestros todavía tienen un largo trayecto de actividad docente de más de 22 años. Pensamos que con la experiencia adquirida que, sobrepasa los quince años en hombres y mujeres, y una capacitación continua se podría potencializar este recurso humano que, como se observará más tarde, emplea un $44 \%$ de estrategias tradicionales en sus clases.

Respecto a los estudios realizados por los docentes, 63,93\% de los profesores posee la titulación universitaria en el área de inglés (Profesor de Segunda Enseñanza en inglés y Licenciados en Ciencias de la Educación en inglés) mientras que el 36,07\% de profesores de inglés no tienen formación en esta área. Llama la atención que una tercera parte de profesores fiscales de esta muestra no tenga una titulación en el área de inglés lo que podría incidir de forma negativa en los educandos al no manejar la lengua extranjera ni la metodología en forma apropiada. Aún más, existe un grupo pequeño 
$(18,56 \%)$ que tiene postgrados; sin embargo, ninguno de estos postgrados se enmarca en la enseñanza del inglés.

De los profesores titulados $(63,93 \%)$, que equivale a 112 docentes, 45 de ellos han vivido en un país anglosajón, mientras que de los 9 profesores sin título (bachilleres), dos de ellos han tenido una residencia temporal en un país de habla inglesa. Quizá esta experiencia de vida en un país extranjero, sea la razón por la cual se les adjudicó una partida fiscal en la cátedra de inglés.

Vale mencionar brevemente las condiciones en las que se realiza el proceso enseñanzaaprendizaje y los sujetos involucrados. Así, los colegios fiscales funcionan en diferentes horarios: matutino, vespertino y nocturno. En muchos casos, el mismo edificio es utilizado en estos tres horarios. Esta sobrecarga de utilización causa problemas en el deterioro del espacio físico. Por otro lado, algunas de estas instituciones son utilizadas para el funcionamiento de escuelas y colegios lo que en muchos casos no brinda las comodidades necesarias a todos los estudiantes; es decir, la misma infraestructura no puede ser utilizada por niños y adolescentes. Pocas de estas instituciones son edificios modernos que cuentan con facilidades de aulas amplias y áreas de recreación. Con respecto a los estudiantes, es bien conocido que los alumnos de las instituciones fiscales, en su mayoría, pertenecen a una clase media y media baja que no cuentan con todos los recursos económicos suficientes que ayuden a un aprendizaje efectivo. Existe un alto número de estudiantes por clase, que también influye en el proceso enseñanza-aprendizaje de manera negativa como se observa en la tabla correspondiente.

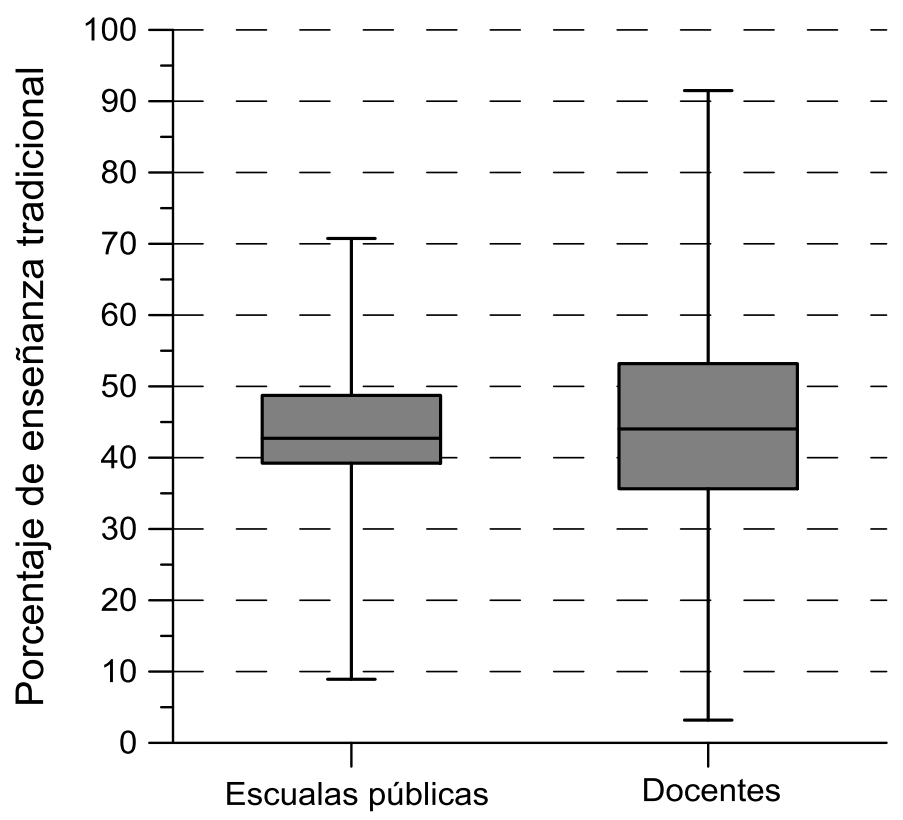

Figura 2. Nivel de uso de estrategias tradicionales en los colegios fiscales y en los docentes.

Analizando los resultados obtenidos sobre las percepciones de enseñanza de los profesores en las encuestas, se observa que el promedio del puntaje de enseñanza tradicional o ATES -Average Traditional Education Score- fue de 0,44, con una desviación estándar de 0,14, lo que implica que en promedio la enseñanza de inglés en los colegios de la ciudad tendría un componente de un $44 \%$ tradicional. Cada colegio presenta un nivel de tradicionalismo determinado, es decir, existen colegios con mayor tendencia tradicionalista que otros (siendo el $9 \%$ el mínimo porcentaje y $70 \%$ el máximo); sin embargo, este componente tradicionalista en la enseñanza del inglés no debe ser confundido con un ranking entre los colegios pues representa, como ya se mencionó, un promedio de enseñanza tradicional por cada colegio.

Como se había indicado, el promedio de la enseñanza de inglés en los colegios de la ciudad de Cuenca tendría un componente de un $44 \%$ tradicional (Figura 2). Individualmente, cada profesor, presenta un determinado porcentaje de utilización de estrategias tradicionales. Se observa un rango 
mayor de estrategias tradicionalistas, por lo tanto existe mayor variación entre los docentes, siendo $45 \%$ el promedio de estrategias tradicionales empleado por los profesores de inglés.

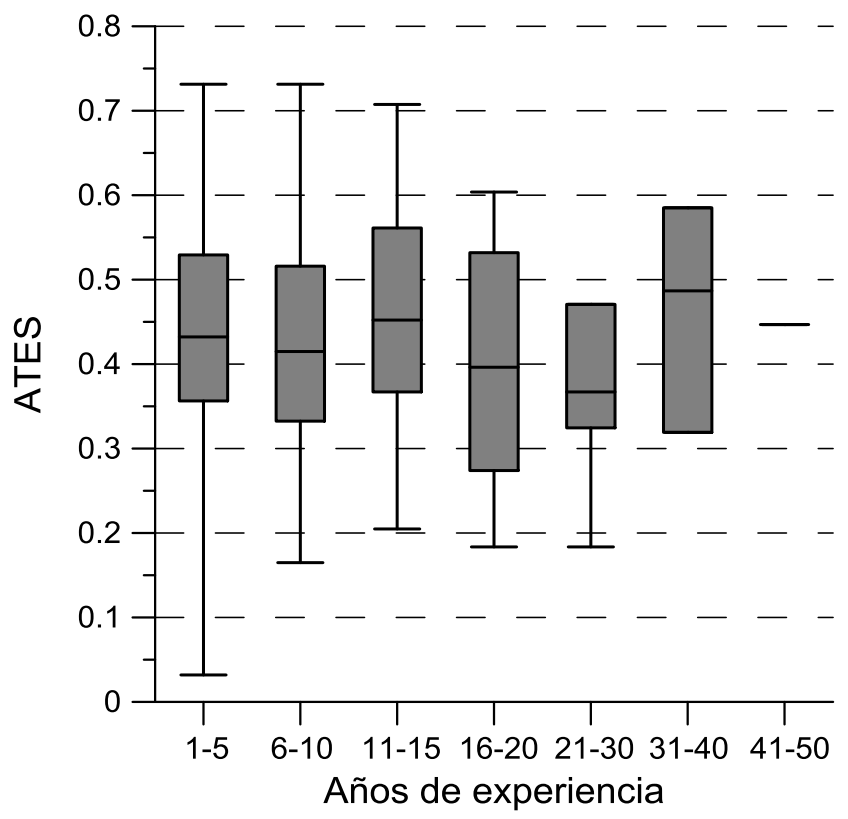

Figura 3. Niveles de metodología tradicional según años de experiencia.

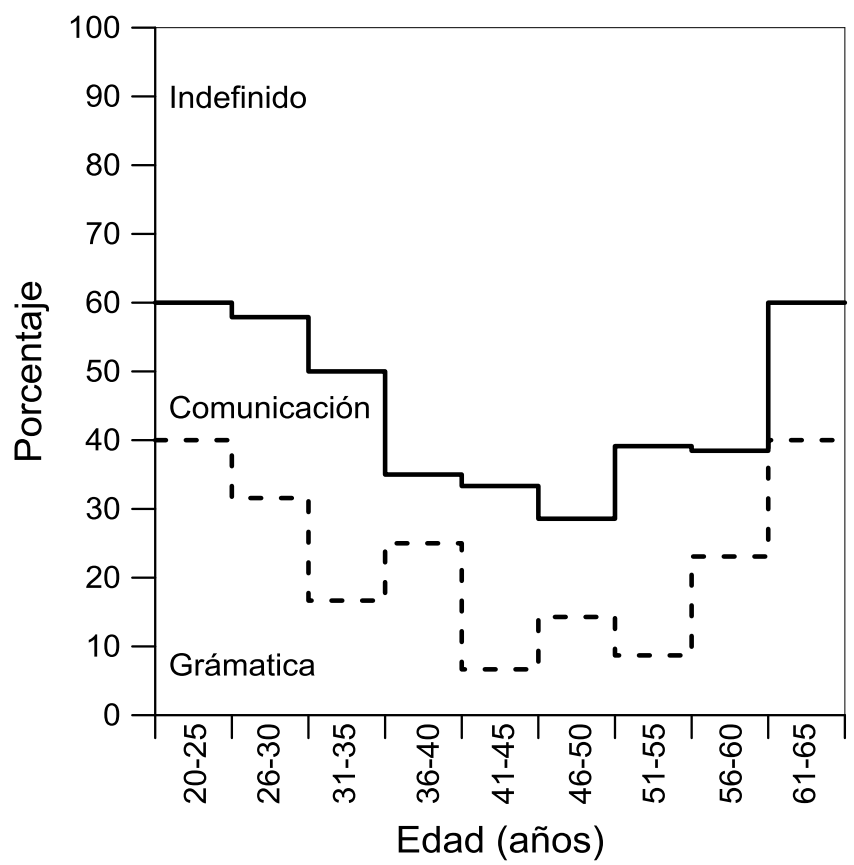

Figura 4. Tendencias en gramática y comunicación según los años de experiencia.

Por otro lado, la encuesta permitió relacionar la variable años de experiencia con la enseñanza tradicional o ATES. La Figura 3 nos muestra esta relación. La variable de los años de experiencia de los docentes respecto al ATES revela que no es un factor determinante en el comportamiento de la didáctica tradicional. De acuerdo a este análisis, se descarta que exista una tendencia significativa; en consecuencia, el ATES promedio no está influenciado por los años de experiencia. Esta correlación nos indica una variabilidad en términos generales; es decir, no existe una diferencia entre los profesores con mayor número de años de experiencia y los profesores jóvenes. Se concluye entonces que los profesores jóvenes no están aplicando nuevas metodologías como podría haberse esperado. 
Sin embargo, se observa que a mayor número de años de experiencia la población docente presenta una menor variabilidad de empleo de estrategias tradicionales y comunicativas.

De acuerdo a la Figura 4, en la que se correlacionan la edad de los profesores con la variable de enseñanza comunicativa en la enseñanza del inglés con un énfasis en la comunicación o en la gramática, se observa que existe una gran variación de acuerdo a las diferentes edades; sin embargo, se nota una tendencia mayor en los primeros años de docencia hacia la comunicación en contraste con la gramática. Esta tendencia disminuye en los siguientes 25 años para luego incrementarse en los profesores de 50 años en adelante. Por otro lado, el énfasis de la enseñanza en la gramática presenta mayor variabilidad con los años de experiencia, pues se observa incrementos y disminuciones en esta directriz -aunque siempre es menor a la comunicativa- para finalmente incrementarse a partir de los 56 años de edad. Cabe anotar que la gran mayoría de profesores encuestados no manifiesta una tendencia específica con respecto a lo comunicativo o a lo gramatical pues su patrón tradicionalista depende de otras variables (didáctica, uso de traducción, limitada interacción, número de estudiantes, entre otros) que clarifican su predisposición.

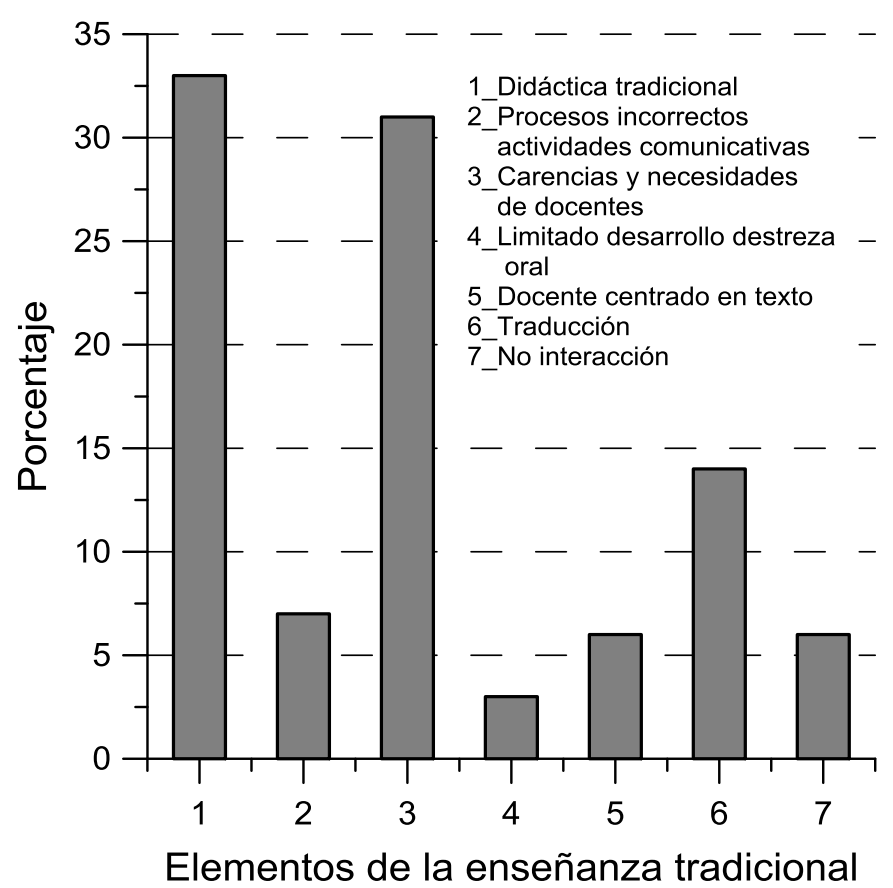

Figura 5. Porcentaje de los elementos constitutivos de la enseñanza tradicional según la entrevista.

La Figura 5 nos señala la existencia de un margen mínimo de profesores (3\%) que manifiesta un limitado desarrollo de la destreza oral en los alumnos; en cambio la mayoría de docentes sostiene que el habla es determinante en el proceso de enseñanza de una lengua. Sin embargo, las observaciones de clases indican que no se promueve el desarrollo de la destreza oral como lo afirman los profesores en la entrevista. Por ejemplo, una actividad importante que desarrolla la comunicación verbal es el diálogo controlado; sin embargo, la observación revela que el $80 \%$ de profesores no utiliza esta actividad, mientras que el $10 \%$ lo hace apropiadamente y un $10 \%$ la aplica de una manera parcial. Del total de la muestra solamente dos profesores utilizaron una actividad significativa conocida como role play que promueve el uso del lenguaje oral de una manera más espontánea, a través de la asignación de roles a los estudiantes dentro de un contexto específico.

Seis por ciento de los docentes manifiesta en la entrevista la existencia de algunas limitaciones respecto al uso de estrategias que promuevan la interacción entre estudiantes. Se esperaría observar, entonces, un número elevado de profesores utilizando estrategias que fomenten la interacción. Sin embargo, las observaciones realizadas reflejan el limitado uso de estrategias y actividades comunicativas. Del total de 92 profesores observados, se distingue que un $84 \%$ de ellos no utiliza una importante actividad comunicativa planteada en muchas tareas de los textos Our World Through 
English, conocida como information gap, en la que dos estudiantes intercambian información que uno de ellos posee y el otro la desconoce, imitando una situación comunicativa que se da en la vida real. Solamente $7 \%$ de profesores utiliza esta estrategia de forma apropiada y 9\% la aplica inapropiadamente, es decir, los docentes intentan utilizar estrategias comunicativas pero no logran aprovechar la actividad en forma efectiva, lo cual puede tener efectos contraproducentes como la confusión y el desinterés por parte de los educandos.

La entrevista nos muestra que existe un número de docentes (6\%) que se centra en el texto al momento de realizar su clase sin requerir otros materiales didácticos. En esta práctica se reitera la utilización mecánica de actividades del libro, lo que las convierte en poco significativas para los estudiantes. La observación nos indica que existe interacción entre el profesor y un número reducido de estudiantes, usualmente con aquellos que se sientan en las primeras filas o con los que tienen mayor dominio de la lengua, constituyéndose esta situación en otra característica de este textocentrismo. El 36\% de profesores mantiene el modelo de interacción profesor-estudiante, relación que se reduce a hacer preguntas y contestarlas, traducir, y escribir en la pizarra bajo la guía del profesor. De ello se deriva la conclusión de que no existe un cambio significativo en lo que a interacción respecta. De acuerdo con Vargas (1995), uno de los factores del bajo nivel de inglés, incluye el empleo de una metodología obsoleta centrada en el profesor. Estos datos coinciden con los reportes de contextos internacionales que señalan que, a pesar del cambio de currículo dentro de un enfoque comunicativo, éste no se lo ejecuta apropiadamente debido al desconocimiento del manejo de actividades comunicativas (Liu, 2004). El empleo de estas estrategias constitutivas de la enseñanza tradicional (limitado desarrollo oral, traducción, texto-centrismo, y no interacción) podría estar relacionado con el desconocimiento del nuevo currículo por parte de los docentes, así como también con la existencia de clases numerosas donde se desenvuelven los docentes, ello confirma los hallazgos realizados en las observaciones. Al respecto Liu (2004) señala que existe una resistencia a prácticas innovadoras de enseñanza debido a la baja competencia comunicativa en la lengua y al gran tamaño de grupos de estudiantes, como ocurre en la educación fiscal en la ciudad de Cuenca.

Respecto al uso de estrategias tradicionales de mayor incidencia, la entrevista indica que un $14 \%$ de profesores hacen uso de la traducción en relación a otros métodos. En la práctica, según la observación, este porcentaje se incrementa a un $73 \%$ de docentes que apelan a esta estrategia en relación a un $27 \%$ que no lo hacen. La traducción es un mecanismo muy generalizado en la enseñanza del inglés, no sólo se traducen las instrucciones, sino las actividades, o el "input". Por ejemplo, los textos no se los utiliza para desarrollar lectura comprensiva sino para traducirlos. Cuarenta y tres punto nueve por ciento de profesores traduce permanentemente frente a $27,4 \%$ que no lo hace, y $28,5 \%$ lo hace parcialmente. En lo que respecta al uso del inglés por parte de los profesores en el aula, la observación muestra que el $41,7 \%$ habla con fluidez, 31,8\% tiene algunos problemas menores, pero el $26,3 \%$ de profesores no son competentes en la lengua extranjera. El $19 \%$ de profesores usan español la mayor parte del tiempo, el $23 \%$ usa la lengua nativa para explicaciones gramaticales, clarificar conceptos o dar instrucciones, mientras que $21 \%$ no la usa. Esta información coincide con Vargas (1995), Criollo y Pulla (2000), quienes observaron hace más de una década la tendencia a utilizar la lengua materna como medio de instrucción.

Un porcentaje significativo de docentes (31\%) expresa tener una serie de necesidades relacionadas al uso de recursos didácticos, capacitación y mejoramiento en el manejo del inglés, incluso dentro de la formación académica de futuros docentes. Las siguientes citas tomadas de la entrevista ilustran esta última afirmación:

$\checkmark$ “... que nos den unos cursitos de metodología más de punta, como quien dice más moderna porque yo, cuando vienen las chicas de la universidad a hacer las prácticas (pedagógicas), yo pensé que estaban más modernizadas y total estaban dando al mismo estilo contemplando la gramática, enfocando la gramática, el vocabulario, entonces dije no he estado tan rezagada ..."

$\checkmark$ “... some handouts or some material because we don't have any material ..."

Una serie de imprecisiones en el manejo de conceptos básicos de la enseñanza del inglés expresados en las entrevistas, demuestran la necesidad de perfeccionamiento en áreas básicas de la formación docente. Por ejemplo: 
$\checkmark$ Los profesores manifiestan confusión entre destrezas y estrategias: "mi estrategia favorita es speaking ..."

$\checkmark$ Confusión entre objetivos con desarrollo de destrezas: "lección está basada en el listening ... y las dos primeras tareas eran de listening ..."

$\checkmark$ Énfasis en objetivos gramaticales: "los objetivos ... de mi clase fueron que ellas diferencien entre como un adjetivo puede transformarse en un sustantivo para escribir oraciones".

La práctica docente observada y las concepciones de los profesores revelan necesidades específicas de capacitación en el área de enseñanza de inglés, así como también la necesidad del planteamiento de objetivos que incluyan un propósito comunicativo y no solamente un énfasis gramatical en las formas lingüísticas. Con respecto a la didáctica tradicional, un 33\% de profesores expresa que emplea variadas actividades propias de la didáctica tradicional. Se destaca el manejo de la gramática descontextualizada donde sobresalen la utilización de tablas para un análisis estructural, la repetición coral, los objetivos de clase centrados en la gramática, así como también el empleo de listas de vocabulario, entre otros. Ejemplo de la conservación de la lista de vocabulario es la siguiente cita: “... al finalizar ellos (los estudiantes) pueden extraer todas las palabras nuevas, todas las palabras nuevas que vieron en esta clase para ... ellos tienen unas 50 páginas para hacer el vocabulario".

A pesar de que las encuestas y entrevistas indican una tendencia a la enseñanza de gramática contextualizada, ésta no se visualiza en las observaciones, pues se constató que la gran mayoría de profesores ni siquiera introducen la gramática en sus clases y, por el contrario, hay pocos profesores que la introducen de manera descontextualizada. Existe un avance en este sentido, pues según Vargas (1995) hasta el año 1995 los docentes de inglés se enfocaban en la gramática como elemento primordial de su sílabo, dictándola fuera de contexto. Es decir, la gramática dejó de ser el centro de la enseñanza de una lengua. Sin embargo, vale señalar, que el proceso se invirtió; una gran mayoría ignora en sus clases la enseñanza de la gramática. Esta situación podría responder al hecho de que en los textos de inglés del estudiante, solamente al final de cada unidad, se incluye contenidos y ejercicios gramaticales que, según las observaciones, no fueron considerados por los profesores. Así también, la ausencia de explicaciones gramaticales podría responder a que los profesores, conscientes de los cambios prioritarios del nuevo currículo, no incluyen gramática en sus clases. La ausencia total de la enseñanza de la gramática podría constituir un obstáculo para el aprendizaje integral de un idioma.

Por otro lado, existe un importante porcentaje de 38\% de docentes que utiliza una didáctica comunicativa que implica el empleo de juegos y otras actividades promoviendo la motivación entre los estudiantes.

\section{CONCLUSIONES Y RECOMENDACIONES}

\subsection{Conclusiones}

Luego de la finalización del análisis y discusión de resultados se llega a las siguientes conclusiones:

$\checkmark$ El tratamiento estadístico y las observaciones contestan la pregunta referente a la identificación del sustento pedagógico que fundamenta las estrategias de enseñanza empleadas por los profesores. La información obtenida indica que las estrategias empleadas por los profesores se enmarcan en un modelo tradicional a pesar de la obligatoriedad del Ministerio de Educación y Cultura del Ecuador de implementar un currículo con un enfoque comunicativo desde el año 1993 a través del Proyecto CRADLE.

$\checkmark$ Dentro de este marco tradicionalista, se observa que aún se mantienen ciertos problemas encontrados antes de la implementación del nuevo currículo como son: clases centradas en el profesor, utilización del método de gramática y traducción y carencia del manejo de la lengua extranjera como medio de instrucción, factores que podrían contribuir al limitado desarrollo del inglés por parte de los estudiantes.

$\checkmark$ Aun cuando se continúa con la utilización de una metodología tradicional, sin embargo, se observa que en la actualidad las clases no se enfocan mayoritariamente en las estructuras del lenguaje, es 
decir en la gramática. Este avance involucra también un aspecto negativo al no incluir ningún elemento gramatical en las clases.

$\checkmark$ Se nota que existe un pequeño número de profesores que utiliza una didáctica más comunicativa enfocada al desarrollo de las destrezas de la lengua. Sin embargo, de este número, algunos docentes no implementan apropiadamente el enfoque comunicativo.

$\checkmark$ Una de las causas del uso de la didáctica comunicativa podría ser la implementación del currículo diseñado por el Ministerio de Educación que provee la serie de textos enmarcada en este enfoque, así como también, a factores externos relacionados con la capacitación de profesores, variables que no han sido analizadas en este estudio.

$\checkmark$ Una de las causas de la tendencia tradicional imperante en nuestras aulas estaría conectada con el elevado número de estudiantes y condiciones físicas inapropiadas que no permiten al profesor proveer de espacios de interacción a los alumnos así como del monitoreo oportuno a los educandos.

\subsection{Recomendaciones}

Se requiere capacitación continua sobre el manejo de nuevas alternativas metodológicas para la enseñanza de la lengua inglesa desde una perspectiva comunicativa a fin de superar el método tradicional que sigue vigente, lo que contribuirá a superar las clases centradas en el profesor, la utilización de traducción y la carencia del manejo de inglés como medio de instrucción. Además, los resultados han demostrado que los profesores jóvenes al igual que los de más edad utilizan una metodología tradicional. Las universidades como corresponsables de la formación de los profesores en la enseñanza comunicativa deberían enfatizarla en la práctica docente, como sustento de la fundamentación teórica. Así también, sería beneficioso resaltar en los nuevos profesores que la gramática es una herramienta que debe ser impartida de una manera comunicativa y en contexto. Se recomienda a los profesores de inglés de los colegios coordinar sus actividades a nivel de área en términos metodológicos, de concatenación de contenidos y criterios de evaluación afines a la metodología comunicativa. Finalmente, los autores sugieren implementar un proyecto de intervención dirigido a la capacitación de los docentes enmarcado en una visión comunicativa. Una alternativa podría ser talleres enfocados en el aprendizaje por tareas o Task-based learning, como se lo conoce en inglés.

\section{AGRADECIMIENTOS}

Agradecemos a los miembros de la Dirección de Investigación de la Universidad de Cuenca por brindarnos el apoyo financiero para la investigación, a las funcionarias del Departamento de Idiomas Extranjeros de la Dirección Provincial de Educación del Azuay, Esp. Rocío Fuertes y Mst. Lorena Arévalo, y a los profesores de los colegios fiscales de la ciudad por permitirnos realizar las encuestas, observaciones y entrevistas. A Cristina Espinel y Rocío Guncay, estudiantes de la Escuela de Lengua y Literatura Inglesa que participaron como ayudantes de investigación. Finalmente, un sincero agradecimiento al Ing. Vladimiro Tobar, profesor de la Facultad de Ingeniería, por su asesoramiento en el análisis estadístico.

\section{BIBLIOGRAFÍA}

Alcón, E., 2004. Research on language and learning: Implications for language teaching. IJES, 4(1), 173-196.

Arévalo, L., 2009. Comunicación personal.

Brown, J.D., T.S. Rodgers, 2004. Doing second language research. Oxford University Press, Oxford, $\mathrm{UK}, 328 \mathrm{pp}$. 
CONCELT, 2004. Evaluación ex-post proyecto CONCELT. Facultad de Filosofía, Letras y Ciencias de la Educación, Universidad de Cuenca, Cuenca, Ecuador, 57 pp.

Criollo, N., J. Pulla, 2000. The impact of the CRADLE project on English teaching in Cuenca's high schools. Tesis previa a la obtención del título de licenciatura en Ciencias de la Educación, Especialidad Inglés. Facultad de Filosofía, Letras y Ciencias de la Educación, Universidad de Cuenca, Cuenca, Ecuador, 361 pp.

Garrido, P., 2007. Foreign language learning in Santo Domingo: Qualitative case studies in two private schools. PhD Dissertation, Department of Educational Policy Studies, College of Education, Georgia State University, GE, USA, 326 pp.

El Mercurio, 2006. Importancia de idiomas. El Mercurio, periódico local de Cuenca. Publicado el 2 de mayo de 2006.

Krashen, S.D., T.D. Terrell, 1983. The natural approach: Language acquisition in the classroom. Pergamon Press, Oxford, UK.

Liu, J., 2004. Methods in the post-methods era. Report on an international survey on language teaching methods. IJES, 4(1), 137-152.

Liyanage, I.J.B., 2004. An exploration of language learning strategies and learner variables of Sri Lankan learners of English as a second language with special reference to their personality types. Unpublished PhD Dissertation, Faculty of Education, Griffith University, Australia, 250 pp.

Luo,W., 2007. English language teaching in Chinese Universities in the era of the World Trade Organization: A learner perspective. PhD Dissertation, School of Global Studies, Social Science and Planning, RMIT University, Melbourne, Australia, 312 pp.

Mackey, A., S.M. Gass, 2005. Second language research. Methodology and design. Lawrence Erlbaum Associates, Inc., Publishers, New Jersey, USA, 424 pp.

McLaughlin, D., 1996. Who is to retrain the teacher trainers? A Papua New Guinea case study. Teach. Teach. Educ., 12(3), 285-301.

Ministerio de Educación y Culturas del Ecuador, 1998. El Proyecto CRADLE. Ministerio de Educación y Culturas del Ecuador, Quito, Ecuador.

Ministerio de Educación y Culturas del Ecuador, 2009. El Proyecto CRADLE. Informe final. Descargado de http://www.lppuerj.net/olped/Mec/cradle/p1.htm el 9 abril 2009.

Ministerio de Educación y Culturas del Ecuador, 2008. Programa Nacional de Inglés. Diseño curricular por competencias. Dirección Nacional de Currículo, División de Idiomas Extranjeros, Ministerio de Educación y Cultura del Ecuador, Quito, Ecuador.

Mohamed, N., 2006. An exploratory study of the interplay between teachers' beliefs, instructional practices and professional development. PhD Dissertation, Department of Language Teaching and Learning. The University of Auckland, Australia, 344 pp.

Richards, J., T. Rodgers, 2001. Approaches and methods in language teaching. Cambridge University Press, Cambridge, UK, 270 pp.

Roberts, J., 2004. The communicative approach to language teaching: The King is dead! Long live the King! IJES, 4(1), 1-37.

Savignon, S., Ch. Wang, 2003. Communicative language teaching in EFL contexts: Learner attitudes and perceptions. IRAL, 41, 223-249.

Shono, S., 2004. Good ESL teaches: From the perspectives of teachers and adult learners. PhD Dissertation, Faculty of the Graduate School, The University of Texas, Austin, TX, USA, 221 pp.

Taylor, P., A. Mulhall, 2001. Linking learning environments through agricultural experience Enhancing the learning process in rural primary schools. Intern. J. Educat. Develop., 21(2), 135148.

Vargas M., 1995.The role of baseline studies in project evaluation - A case study. Unpublished MA (TEFL: Teaching of English as a Foreign Language) dissertation. The Centre for Applied Language Studies, The University of Reading, Reading, UK, 145 pp. 
MASKANA, Vol. 3, No. 2, 2012

Wang, W., 2008. Teaching English to young learners in Taiwan: Issues relating to teaching, teacher education, teaching materials and teacher perspectives. PhD Dissertation, Department of General and Applied Linguistics, The University of Waikato, Hamilton, New Zealand, 490 pp. 